\title{
Inhibition of return with rapid serial shifts of attention: Implications for memory and visual search
}

\author{
MICHAEL D. DODD, ALAN D. CASTEL, and JAY PRATT \\ University of Toronto, Toronto, Ontario, Canada
}

\begin{abstract}
Horowitz and Wolfe (2001) suggested that inhibition of return (IOR) should not be observed in tasks that involve rapid deployments of attention. To examine this issue, five of six possible locations were sequentially cued with either short-duration peripheral cues $(50 \mathrm{msec})$ or long-duration peripheral cues $(500 \mathrm{msec})$. As was expected, IOR was observed in the first two experiments at every cued location with the long-duration cues, with the magnitude of IOR decreasing for earlier cued locations relative to later cued locations. In the short-cue condition, IOR was observed at only one cued location (the second to last). The pattern of results for the short-duration cues was found regardless of whether the fixation cue was of a short (Experiment 1) or a long (Experiment 2) duration. In Experiment 3, the final fixation cue was removed, and IOR was again observed at virtually all locations in both the short- and the long-cue conditions. These findings indicate that IOR can be observed at multiple locations when attention is shifted rapidly between locations.
\end{abstract}

Allocating attention to salient features in the visual environment is a critical function of the visual system. One process that is thought to facilitate the acquisition of visual information is inhibition of return (IOR). IOR refers to the finding that targets at cued locations are more slowly responded to than targets at uncued locations when a relatively long temporal interval (typically $200 \mathrm{msec}$ or greater) intervenes between the two events (e.g., Posner \& Cohen, 1984; for a recent review, see Klein, 2000). Since the initial discovery of IOR, the inhibitory effect has often been thought of as a process that facilitates visual search by preventing attention from returning to previously attended locations (e.g., Klein, 1988; Pratt, Kingstone, \& Khoe, 1997; Tipper, Weaver, Jerreat, \& Burak, 1994). In order to make searches more efficient, IOR must involve a memory component that is used to keep track of where attention has been, to ensure that attention does not return there (e.g., Klein, 1988).

To determine whether IOR is involved in visual searches, Klein (1988) had participants perform one of two different search tasks (an easy parallel search or a difficult serial search), followed by a luminance detection task in which the probes could appear at locations

This research was supported by a Natural Sciences and Engineering Research Council (NSERC) grant to J.P. and by NSERC postgraduate scholarships to M.D.D. and A.D.C. We thank Meira Lewis for collecting the data. We appreciate the comments of Yuji Takeda and two anonymous reviewers on previous versions of this article. Correspondence regarding this article may be sent to M. D. Dodd, A. D. Castel, or J. Pratt, Department of Psychology, 100 St. George Street, University of Toronto, Toronto, ON, M52 3G3 Canada (e-mail: michaeld@psych.utoronto.ca, alan@psych.utoronto.ca,or pratt@psych.utoronto.ca). where an item had appeared in the previous search task (on-probes), or at a location where an item had not appeared (off-probes). On the basis of the work of Treisman (1986; Treisman \& Gelade, 1980), Klein (1988) reasoned that the serial search task condition should produce longer detection times for on-probes, relative to offprobes, if the participants serially attended to each item. The previously attended locations would then be tagged for subsequent inhibition. In contrast, no such difference between on-probes and off-probes was expected for the parallel search condition, since the presence or absence of the targets could be identified preattentively. This would lead to no previously attended locations and, therefore, no subsequent inhibition. Klein's (1988) results were consistent with these predictions, with IOR found for on-probes in the serial condition and not in the parallel condition. Although this result initially failed to be replicated (Wolfe \& Pokorny, 1990), it has recently been demonstrated that serial on-probes are more slowly detected than serial off-probes when the search display from the initial task remain visible until the luminance probe is detected (moreover, the response time [RT] difference between on-probes and off-probes is larger in a serial search condition than in a parallel search condition; Müller \& von Mühlenen, 2000; Takeda \& Yagi, 2000). These results provide support for a memory-based model of IOR, in which previously attended locations are tagged and placed in memory so that attention does not return to these locations.

More evidence that IOR involves a memory component comes from studies that have shown that IOR occurs at several sequentially cued locations (e.g., Danziger, Kingstone, \& Snyder, 1998; Snyder \& Kingstone, 2000, 
2001). Although it was initially unclear whether IOR could exist at more than one cued location (Abrams \& Pratt, 1996; Pratt \& Abrams, 1995; Tipper, Weaver, \& Watson, 1996), there is now strong evidence that several sequentially cued locations can be inhibited (Danziger et al., 1998; Snyder \& Kingstone, 2000). For example, Danziger et al. used a visual display consisting of five peripheral placeholders with up to three sequential peripheral cues and observed IOR at every cued position. Furthermore, the magnitude of IOR was greater for the more recently cued locations (i.e., cues that appeared closer in time to the target) than for earlier cued locations.

To further determine the number of locations that could be simultaneously inhibited, Snyder and Kingstone (2000) expanded Danziger et al.'s (1998) paradigm by increasing the number of possible target locations, as well as the number of cues. Using eight position placeholders and six sequential cues (with a duration of $500 \mathrm{msec}$ for each cue), Snyder and Kingstone (2000) observed IOR at the five most recently cued locations, with only the initially cued location not showing a reliable IOR effect. Again, the magnitude of IOR decreased as a function of when the cue occurred, with greater IOR effects observed at more recently cued locations than at earlier cued locations. Although the results of Kingstone and colleagues are consistent with a role for IOR in visual search (i.e., there is memory for several previously attended locations), they also suggest that this system has a limited capacity, since the magnitude of IOR decays in a generally linear fashion as further positions are cued and is limited to five or six locations.

Although the aforementioned research indicates that there is likely a role for IOR in visual search and that IOR has some type of memory component, Horowitz and Wolfe $(1998,2001)$ have recently proposed that visual search is memory free. Memory-free search refers to situations in which one does not remember previously attended locations, so that previous deployments of attention do not influence where attention is directed later in time. Horowitz and Wolfe (2001) used a multiple-target search paradigm and posited that search time should increase linearly as the number of targets to search for increased (the task being a greater than/less than judgment on the number of targets) if visual search contained a memory component that tagged already visited locations. If, however, visual search was memory free, they predicted an accelerating RT function, indicating that nontarget locations were being revisited during search. Their results were consistent with the latter prediction, prompting them to make the following statement concerning IOR and the "inhibitory tagging function" that is thought to cause it:

IOR takes $300 \mathrm{msec}$ to overcome the initial facilitation at an attended location (Posner \& Cohen, 1984). If attention is redeployed to a new stimulus every 25,50 , or even $100 \mathrm{msec}$, then IOR will not have time to influence the selection of the next item, though it could play a role in deployments of the eyes or of attention in long searches with large set sizes.

(Horowitz \& Wolfe, 2001, p. 280)
Essentially, Horowitz and Wolfe (2001) suggest that IOR should not accrue to any location that has been attended for less than $100 \mathrm{msec}$. Rapidly shifting attention to a new location should prevent a previously attended location from being tagged with inhibition, thus eliminating any role of IOR in a visual search task.

Although the results of Kingstone and colleagues demonstrate that IOR can occur at multiple locations when attention is shifted relatively slowly between items (500 msec per location), there is currently no information regarding Horowitz and Wolfe's (2001) prediction that IOR should not be observed when attention is rapidly shifted between several items. In other words, their statement that IOR will be found with slow deployments of attention is a statement of fact (e.g., Danziger et al., 1998; Snyder \& Kingstone, 2000), whereas their statement that IOR will not be found with fast deployments of attention is a hypothesis. To directly address this hypothesis, in the present study, we employed a multiple-cue methodology similar to that of Snyder and Kingstone (2000), with cues of either short (50 msec) or long (500 msec) duration. On the one hand, if IOR can occur in a setting where locations are rapidly cued, we should observe IOR with short cues that require attention to be shifted every $50 \mathrm{msec}$. On the other hand, if IOR occurs only with relatively slow deployments of attention, IOR for multiple locations should be found only with the 500-msec cues (replicating the findings from previous studies).

\section{EXPERIMENT 1}

To address the question as to whether IOR can occur when attention is shifted rapidly to new locations, in Experiment 1 , we directly compared the IOR found at multiple sequentially cued locations with short- $(50 \mathrm{msec})$ and long- (500 msec) duration cues. Similar to Snyder and Kingstone's (2000) study, the display consisted of six position placeholders, five of which were cued in a random sequential order on each trial. The use of 50-msec cues allowed us both to approximate the speed at which attention moves in a normal visual search and to test Horowitz and Wolfe's (2001) prediction that IOR should not accrue to locations that have been attended for less than $100 \mathrm{msec}$. On the basis of the results of Snyder and Kingstone (2000), we predicted that significant IOR would be found with the long-duration cues. Furthermore, the amount of IOR should decrease as a function of cue position, with the greatest amount of IOR occurring in the last cued position (Cue 5), whereas the smallest amount of IOR should occur in the first cued position (Cue 1). Finally, if Horowitz and Wolfe (2001) are correct in suggesting that IOR will not occur when items are rapidly scanned, IOR should not be observed with the short-duration cues, because attention should not remain at any one location long enough for that location to be tagged with inhibition.

\section{Method}

Participants. Seventeen undergraduate students from the University of Toronto volunteered to participate in the experiment and 
received course credit for their participation. All the students had normal or corrected-to-normal vision and were naive as to the purpose of the experiment, which took place in a single 1-h session.

Apparatus and Procedure. The experiment was conducted on a 486 PC with VGA monitor in a dimly lit, sound-attenuated testing room. The participants were seated $44 \mathrm{~cm}$ from the front of the computer monitor, with their heads held steady by a chin- and headrest. A keyboard was placed directly in front of the participants, and they made responses by using the space bar on the keyboard.

At the beginning of each trial, a central fixation point (white, $0.2^{\circ}$ in diameter) and an experimental display consisting of a circular arrangement (the diameter of which was $10^{\circ}$ ) of six white outline square placeholders (each subtending $1.0^{\circ}$ and equally spaced along an imaginary circle) was presented on the computer monitor with a black background (see Figure 1).

The participants were instructed to fixate on the central fixation point and not to make any eye movements. After a period of
$500 \mathrm{msec}$, one of two cuing sequences was initiated. In the long-cue condition, five of the six squares were cued sequentially by placing a white circle (subtending $0.8^{\circ}$ ) inside the placeholder for a period of $500 \mathrm{msec}$. The onset of each subsequent cue began immediately after the offset of the cue that had preceded it. The cue locations were randomized on each trial, with the limitation that a location could be cued only once per trial. The participants were explicitly informed that the cues were not predictive of the location of the upcoming target. Following the offset of the fifth cue, the central fixation point was cued for a period of $500 \mathrm{msec}$, at the offset of which a target (a white square that filled the entire placeholder) appeared immediately inside one of the six placeholders on the screen. The target was equally likely to appear inside any of the six placeholders. The shortcue procedure was identical to the long-cue procedure, except that the duration of each cue (including the fixation cue) was $50 \mathrm{msec}$.

The participants were instructed to press the space bar as soon as they detected the target and were told to respond as quickly and ac-

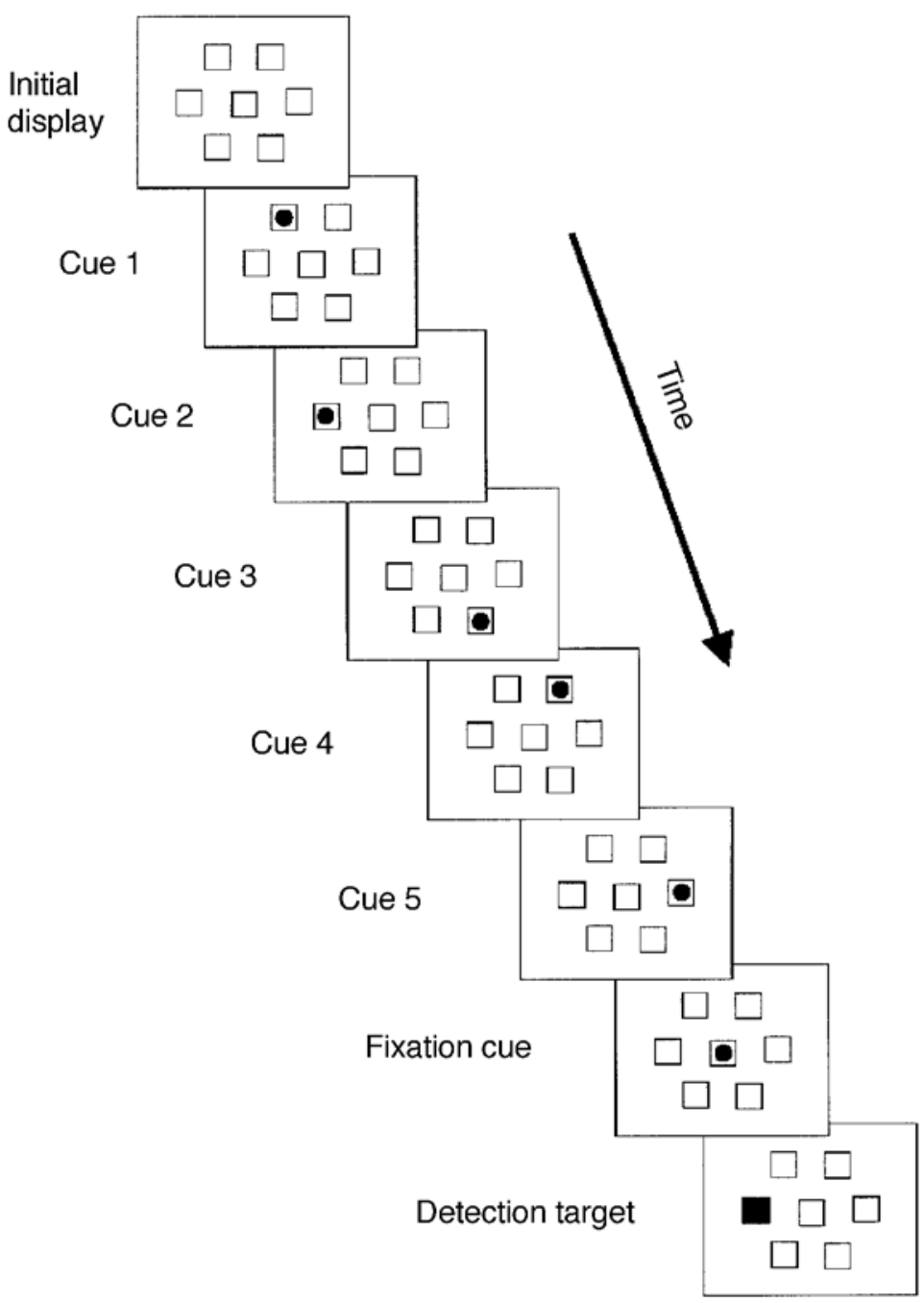

Figure 1. Sequence used in Experiments 1 and 2. The initial display was on for $500 \mathrm{msec}$, and the five cues were presented for either 500 or $50 \mathrm{msec}$ each. In Experiment 1, the fixation cue was presented for $50 \mathrm{msec}$ with the $50-\mathrm{msec}$ peripheral cues and for $500 \mathrm{msec}$ with the 500 -msec peripheral cues. In Experiment 2 , the fixation cue was presented for $50 \mathrm{msec}$ with the $500-\mathrm{msec}$ peripheral cues and for $950 \mathrm{msec}$ with the 50 -msec peripheral cues. See the text for details. 
curately as possible. To reduce anticipatory responses, catch trials in which the target did not appear were also included. The participants were told not to respond if the target did not appear. Incorrect responses on catch trials (and responses less than $100 \mathrm{msec}$ or greater than $1,000 \mathrm{msec}$ ) were considered errors, and a short error tone was presented if any of these occurred. The next trial began $1,000 \mathrm{msec}$ after each response.

Design. The experiment consisted of 560 trials, with 420 test trials and 140 catch trials. Short-duration and long-duration cue sequences were presented in a random order and were equally likely to occur. Short breaks were given after every 112 trials.

\section{Results and Discussion}

Errors occurred on fewer than $0.6 \%$ of all the trials, and these trials were excluded from the analyses. RTs for targets appearing at each location are presented in Table 1. The RTs for targets appearing at each individual cued position were collapsed across trials, as were the RTs for targets appearing at an uncued position.

To examine the IOR $\times$ position effects, the mean RTs were analyzed with a 2 (cue condition:long vs. short) $\times 6$ (target position: uncued position, first cued position, second cued position, third cued position, fourth cued position, or last cued position) analysis of variance (ANOVA). There was a significant main effect of cue condition $\left[F(1,16)=57.59, M S_{\mathrm{e}}=1,001.38, p<.0001\right]$, with faster RTs for targets in the short-cue condition than for targets in the long-cue condition. In addition, there was a significant main effect of target position $[F(5,80)=$ 7.63, $\left.M S_{\mathrm{e}}=155.60, p<.0001\right]$, with longer RTs observed for targets appearing at recently cued locations than for targets appearing at earlier cued locations or at the uncued location, and a significant interaction between cue condition and target position $[F(5,80)=2.61$, $\left.M S_{\mathrm{e}}=183.13, p<.05\right]$.

The total amount of IOR as a function of cue position can be seen graphically in Figure 2. In the long-cue condition, significant IOR was observed at every cue position, and the amount of IOR was greater for late-cued positions than for early-cued positions (all $p \mathrm{~s}<.05$ ). This result is similar to that reported by Snyder and Kingstone (2000) and is consistent with the claim that the magnitude of IOR decreases as the stimulus onset asynchrony (SOA) between cue and target increases (with less IOR observed for early-cued locations than for late-cued locations).

In the short-cue condition, IOR was not observed in four of the five cue conditions, consistent with the sug- gestion of Horowitz and Wolfe (2001). There was, however, a small but significant amount of IOR observed at the second to last cued position [ $8 \mathrm{msec}$, Cue $4 ; t(16)=$ $\left.2.21, M S_{\mathrm{e}}=3.83, p<.05\right]$. This finding is inconsistent with the claim that IOR should not occur when position placeholders are scanned at a rapid pace. Assuming that IOR requires some sort of memory component to tag inhibited locations, this also suggests that there may be a limited role for memory in visual search. Surprisingly, IOR was observed at the second to last cued position, but not at the last cued position in the short-cue condition. There are a couple of reasons why this might have occurred. One possibility is that there was not sufficient time to withdraw attention from the cued location in order for IOR to be generated. This would have resulted in a facilitation effect, however, at the last cued location, something that was not found in the experiment. Another possibility is that the short duration of the cue in the short-cue condition did not allow individuals enough time to tag this location with inhibition. In Experiment 2, we examined this possibility by altering the SOA between the offset of the last cue and the onset of the target in both cue conditions. Regardless, the present results provide preliminary evidence that IOR can occur at locations that have been attended for less than $100 \mathrm{msec}$, contrary to the prediction of Horowitz and Wolfe (2001), who suggest that IOR should not occur when IOR is rapidly shifted from location to location. More specifically, the results of Experiment 1 suggest that IOR can accrue to a location that has been attended for only $50 \mathrm{msec}$.

\section{EXPERIMENT 2}

Although IOR was observed only in one location in the short-cue condition in Experiment 1, it was clear that the overall pattern of results between the long- and shortcue conditions was very similar, with the exception of IOR at Cue 5 (the last cued position). Although Cue 5 elicited the most IOR in the long-cue condition with a decrease in the amount of IOR for subsequently earliercued positions (as would be predicted), no significant IOR was observed at Cue 5 in the short-cue condition. There was, however, a significant amount of IOR observed at Cue 4, with a trend toward a decrease (albeit not significant) in the amount of IOR for subsequently

Table 1

Mean Response Times (in Milliseconds) for Targets Appearing at Each Possible Location in Experiments 1 and 2

\begin{tabular}{cccccc}
\hline \multirow{2}{*}{$\begin{array}{c}\text { Cue } \\
\text { Number }\end{array}$} & \multicolumn{2}{c}{ Experiment 1} & & \multicolumn{2}{c}{ Experiment 2 } \\
\cline { 2 - 3 } \cline { 5 - 6 } & 500 -msec Cues & 50 -msec Cues & & 500 -msec Cues & 50-msec Cues \\
\hline 1 & 394 & 360 & & 402 & 384 \\
2 & 395 & 363 & & 408 & 388 \\
3 & 398 & 364 & & 413 & 388 \\
4 & 403 & 370 & & 415 & 397 \\
5 & 410 & 365 & & 421 & 384 \\
Uncued & 383 & 361 & & 387 & 387 \\
\hline
\end{tabular}




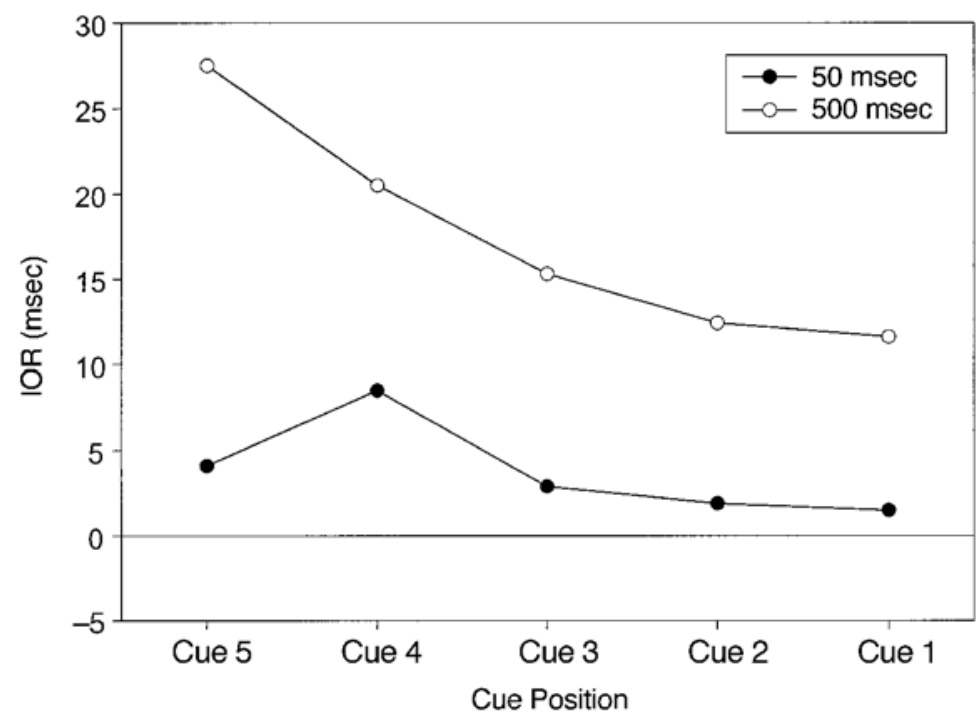

Figure 2. Amount of inhibition of return (IOR, in milliseconds) as a function of each cued position (calculated by subtracting the mean response time [RT] of each cued position from the mean RT of the uncued position) in Experiment 1.

earlier cued positions. This may have been because the brief central fixation cue did not provide enough time for the last cued position to be tagged with inhibition. If it takes some amount of time (greater than $50 \mathrm{msec}$ after the onset of a cue) to tag a location as inhibited, there may be a lag of IOR with regard to the target and the last cued locations. For example, at the moment before the target appears, attention is currently at the fixation location, and IOR has not yet tagged the Cue 5 location but has completed the tagging of the Cue 4 location. Thus, IOR was observed in the fourth cued location, but not in the fifth. In Experiment 2, we directly examined this notion by extending the duration of the fixation cue in the short-cue condition and reducing the duration of the fixation cue in the long-cue condition. If this possibility is correct, IOR should be found at the Cue 5 location in the short-cue condition, because there is now enough time during the long fixation cue to tag the last cued location. It is worth noting that Horowitz and Wolfe (2001) would again predict no IOR at any cued location in the short condition, because no peripheral location was attended for more than $50 \mathrm{msec}$. Finally, the brief fixation cue in the long-cue condition should not affect the pattern of RTs, because each location is attended to for $500 \mathrm{msec}$, which should allow enough time for all locations to be attended to and subsequently inhibited.

\section{Method}

Participants. Seventeen undergraduate students from the University of Toronto volunteered to participate in the experiment and received course credit for their participation. All the students had normal or corrected-to-normal vision and were naive as to the purpose of the experiment, which took place in a single 1-h session. None had participated in the previous experiment.

Apparatus, Procedure, and Design. The apparatus, procedure, and design were the same as those in Experiment 1, with the sole ex- ception that the duration of the fixation cue was altered. In the longcue condition, the duration of the fixation cue was reduced from 500 to $50 \mathrm{msec}$, whereas the fixation cue in the short-cue condition was increased from 50 to $950 \mathrm{msec}$. Thus, the SOA between the onset of the final cue and the onset of the target in the short-cue condition was identical to the SOA between the onset of the final cue and the onset of the target in the long-cue condition in Experiment 1.

\section{Results and Discussion}

Errors occurred on fewer than $0.75 \%$ of all the trials, and error trials were excluded from the analyses. RTs are presented in Table 1, and the amount of IOR as a function of each cued position is presented graphically in Figure 3. As in Experiment 1, the RTs for targets appearing at each individual cued position were collapsed across trials, as were the RTs for targets appearing at an uncued position.

The mean RTs were analyzed with a 2 (cue condition: long vs. short $) \times 6$ (target position) ANOVA to determine whether there was an interaction between cue condition and target position. As in Experiment 1, there was a significant main effect of cue condition $[F(1,16)=$ $\left.10.71, M S_{\mathrm{e}}=2,183.00, p<.001\right]$, a significant main effect of target position $\left[F(5,80)=6.95, M S_{\mathrm{e}}=138.40, p<\right.$ $.0001]$, and a significant interaction between the two $\left[F(5,80)=3.84, M S_{\mathrm{e}}=177.25, p<.01\right]$.

As in Experiment 1, in the long-cue condition, significant IOR was observed at every cue position, and the amount of IOR was greater for late-cued positions than for early-cued positions (all $p$ s $<.05$ ). In the short-cue condition, IOR was not observed at four of the five cue locations; however, a small but significant amount of IOR was again observed at the second to last cued position [11 msec, Cue $4 ; t(16)=3.39, M S_{\mathrm{e}}=3.02, p<.01$ ] Thus, even with a long SOA between the offset of the final cue and the onset of the target, IOR was not ob- 


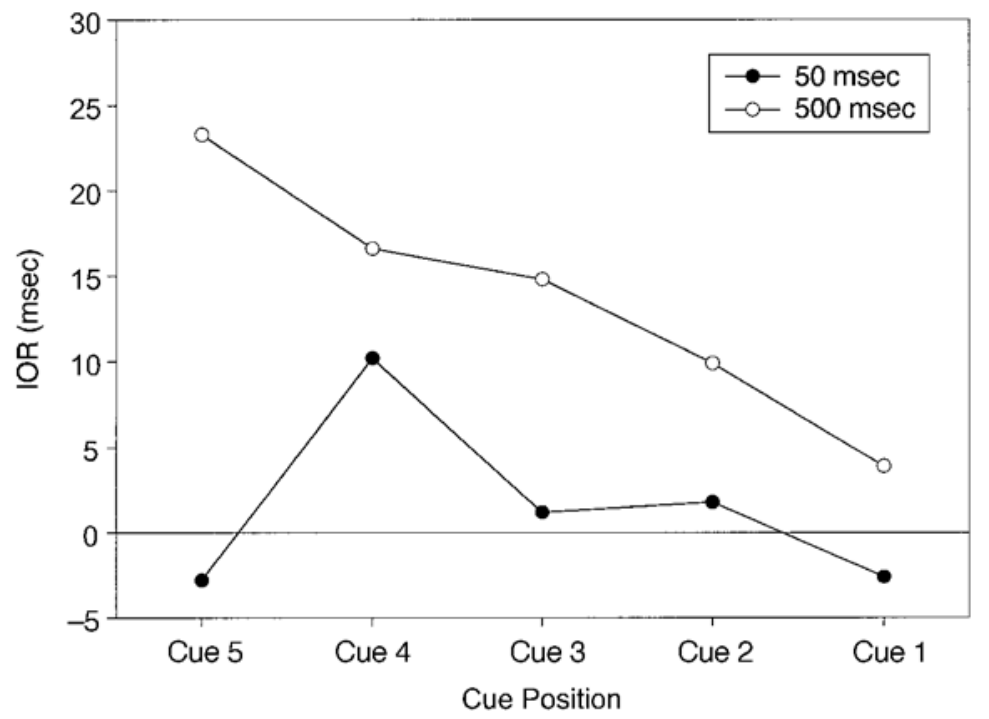

Figure 3. Amount of inhibition of return (IOR, in milliseconds) as a function of each cued position (calculated by subtracting the mean response time [RT] of each cued position from the mean RT of the uncued position) in Experiment 2.

served in the last cued position. This suggests that the observed effect in Experiment 1 was not due to time constraints on the tagging process of the last cued position or to the withdrawal of attention from the cued location.

Unexpectedly, even with a relatively long fixation cue, the short-cue condition replicated the results of Experiment 1 by finding IOR only at the Cue 4 location. Thus, regardless of whether there was $100 \mathrm{msec}$ between the onset of Cue 5 and the target or $1,000 \mathrm{msec}$, IOR was found only at the Cue 4 location. Despite the disparate final SOAs, there is one important similarity between the two experiments: the time interval between the onset of Cue 5 and the onset of the fixation cue. In both cases, the last peripheral cue appeared $50 \mathrm{msec}$ before the fixation cue. Given that the duration of the fixation cue had no effect on the pattern of results, perhaps the critical variable for finding IOR at the last cued location in the short-cue condition was the amount of time between the onset of the last peripheral cue and the onset of the fixation cue. To test this possibility, a third experiment was conducted.

\section{EXPERIMENT 3}

The third experiment was very similar to Experiment 2, except for three important differences. The first was that an eye-monitoring device was used to ensure that the participants retained fixation throughout each trial. Because of the extra time associated with the use of eye-monitoring equipment, separate groups of participants completed the short-cue and the long-cue conditions, whereas in the previous experiments a within-subjects design had been used. The final and most critical difference was that the fixation cue was removed from the trial sequence and re- placed with a delay period. Now, in both conditions, there was a 1,000-msec delay between the onsets of Cue 5 and the target. Similar to the previous experiments, Horowitz and Wolfe (2001) would predict IOR at several locations in the long-cue condition but only at the last cued location in the short-cue condition. This is because, in the short-cue condition, only the Cue 5 location is attended to for more than $100 \mathrm{msec}$, whereas the other four locations are attended to only for $50 \mathrm{msec}$. If the appearance of the fixation cue $50 \mathrm{msec}$ after the last peripheral cue produced the pattern of results found in the previous two experiments, the absence of the fixation cue in the present experiment might produce a pattern more similar to that for the long-cue condition.

\section{Method}

Participants. Thirty undergraduate students from the University of Toronto volunteered to participate in the experiment and received course credit for their participation. All the students had normal vision and were naive as to the purpose of the experiment, which took place in a single 1-h session. None had participated in the previous experiments.

Apparatus and Procedure. The apparatus and procedure of Experiment 3 were similar to those in the first two experiments, but with three key modifications. First, eye movements were monitored to ensure that attention was being captured only by the peripheral cues. Second, due to the extra time associated with the use of eyemonitoring equipment, the experimental conditions (50-msec vs. 500 -msec cues) were now run between subjects, as opposed to within (15 participants in each condition). Finally, the fixation cue was not present after the final peripheral cue (the fixation point, however, remained intact). Thus, in the long-cue condition, there was a $500-\mathrm{msec}$ SOA between the offset of the final cue and the onset of the target (as in Experiment 1), whereas in the 50-msec condition, there was a 950-msec SOA between the offset of the final cue and the onset of the target (as in Experiment 2). This was done to equate for the final cue-target SOA between the two cuing con- 
ditions. In addition, this allowed for a direct comparison between the short-cue conditions in the present experiment and that in Experiment 3 , since the only difference between the two was the presence (Experiment 2) or absence (present experiment) of the fixation cue.

Eye movement monitoring. The eye movement monitor (ASL 210) was calibrated at the beginning of each session, and the calibration was checked at the beginning of each trial. The participants' eye positions were monitored at three different times during the trial (after the onset of the cue at one of the target locations, after the onset of the second cue, and after the onset of the target). If the participant's eyes were more than $1.0^{\circ}$ from the fixation location (along the horizontal axis) at any of these monitored times during a trial, an error tone was emitted, and as with keypress errors, the trial was eliminated from the analysis.

Design. Each experimental condition consisted of 280 trials, with 210 test trials and 70 catch trials. A short break was given after 140 trials.

\section{Results and Discussion}

Eye movement errors occurred on fewer than $1.7 \%$ of all the trials, and other errors (e.g., anticipatory response) occurred on fewer than $0.3 \%$ of all the trials. All error trials were excluded from the analyses. RTs are presented in Table 2, and the amount of IOR as a function of each cued position is presented graphically in Figure 4. As in the previous experiments, the RTs for targets appearing at each individual cued position were collapsed across trials, as were the RTs for targets appearing at an uncued position.

The mean RTs were analyzed with a 2 (cue condition: long vs. short) $\times 6$ (target position) ANOVA to determine whether there was an interaction between cue condition and target position. As in Experiment 1, there was a significant main effect of target position $[F(5,140)=5.16$, $\left.M S_{\mathrm{e}}=353.66, p<.0001\right]$. There was, however, no main effect of cue type $\left[F(1,28)=0.174, M S_{\mathrm{e}}=20,183.97, p=\right.$ .68], nor was there a significant interaction between the two $\left[F(5,140)=0.324, M S_{\mathrm{e}}=353.66, p=.90\right]$. Unlike the first two experiments, IOR was now observed at every location in both the short- and the long-cue conditions.

As in the previous experiments, we examined the amount of IOR as a function of cue position. Again, in the long-cue condition, significant IOR was observed at every cue position, and the amount of IOR was generally greater for late-cued positions than for early-cued positions (all $p \mathrm{~s}<.04$ ). In the short-cue condition, significant IOR was now observed at four of the five cue locations (only the first cued location did not elicit signif-

Table 2

Mean Response Times (in Milliseconds) for Targets Appearing at Each Possible Location in Experiment 3

\begin{tabular}{ccc}
\hline Cue Number & 500-msec Cues & 50-msec Cues \\
\hline 1 & 381 & 372 \\
2 & 385 & 375 \\
3 & 383 & 378 \\
4 & 389 & 375 \\
5 & 392 & 380 \\
Uncued & 365 & 362 \\
\hline
\end{tabular}

icant IOR, although the trend was in the appropriate direction; all other $p \mathrm{~s}<.04)$. Thus, the removal of the fixation cue led to a pattern of IOR in the short-cue condition that was strikingly similar to that observed in the long-cue condition, presumably because the participants were now forced to attend to all of the peripheral cues. This suggests that the pattern of IOR observed in the short-cue conditions of the first two experiments was influenced solely by the presence of the fixation cue. In terms of the issue at hand, this is also the first demonstration that multiple locations can be inhibited after several very rapid shifts of attention.

\section{GENERAL DISCUSSION}

The present experiments demonstrate that IOR can occur in a target detection task when attention is rapidly shifted to multiple locations. In a series of articles, Horowitz and Wolfe $(1998,2001)$ advanced a memory-free model of visual search and made a number of specific comments regarding the role of IOR in visual search. Most important to the present line of research, Horowitz and Wolfe (2001) suggested that IOR should not be observed in tasks that require rapid deployments of attention but, rather, only when sufficient time is given for IOR to develop. Thus, according to Horowitz and Wolfe (1998), any location that is attended for less than $100 \mathrm{msec}$ should not be tagged with inhibition, because inhibition should not have time to accrue. The present experiments directly tested this hypothesis, using a variation of Snyder and Kingstone's (2000) multiple-cuing paradigm. Using cue durations of 50 and $500 \mathrm{msec}$, we were able to test whether IOR would occur with rapid shifts of attention or whether IOR would occur only with relatively slow shifts of attention. Assuming that IOR is involved in visual search and that IOR has a memory component, Horowitz and Wolfe's (2001) model would suggest that we should observe IOR only with 500-msec cue durations, as per the results of Snyder and Kingstone (2000).

In each of the present experiments, IOR was observed at five separate cued locations with cue durations of $500 \mathrm{msec}$. Furthermore, the amount of IOR decreased as a function of cue position, with greater IOR occurring in more recently cued locations. These findings were consistent with those of Danziger et al. (1998) and Snyder and Kingstone (2000), suggesting that IOR decays with time or with the number of intervening cues (it is worth noting that the issue of time vs. intervening items has yet to be resolved). When 50-msec cues were used and were followed by a reorienting cue to fixation, IOR was not observed at four of the five cued locations (Experiments 1 and 2), as would be expected if rapid shifts of attention between locations prevents IOR from influencing the selection of subsequent locations. There was, however, a small but significant amount of IOR observed at the second to last cued position with the 50-msec cues. The pattern of results observed was independent of whether the duration of the fixation cue was short (Ex- 


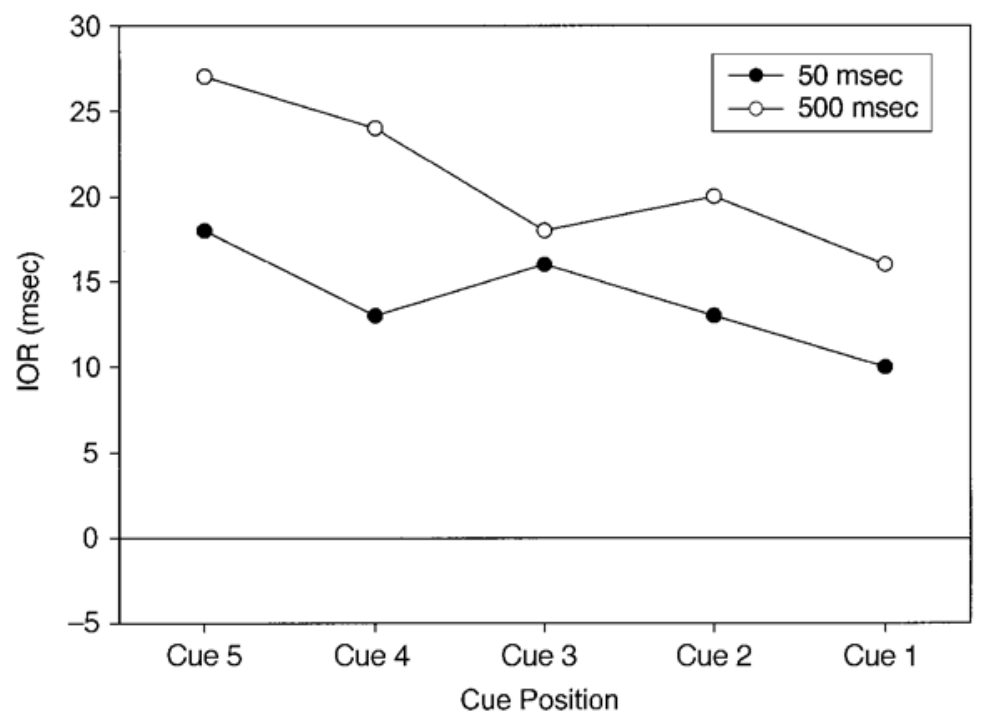

Figure 4. Amount of inhibition of return (IOR, in milliseconds) as a function of each cued position (calculated by subtracting the mean response time [RT] of each cued position from the mean RT of the uncued position) in Experiment 3.

periment 1) or long (Experiment 2). In Experiment 3, however, we removed the fixation cue and observed substantial IOR at four of the five cued locations with 50 -msec cues. The presence of IOR in the short-cue condition is inconsistent with Horowitz and Wolfe's (2001) suggestion that IOR should not be observed when attention is shifted rapidly between locations "every 25,50 , or even $100 \mathrm{msec}$ " (p. 280). Although Danziger et al. (1998) and Snyder and Kingstone (2000) had already demonstrated that IOR is observed in multiple locations with long-duration cues, the present results are particularly important, since they are the first demonstration that IOR can be observed at multiple locations following very rapid cues.

It is worth noting that Experiment 1 is the most direct test of Horowitz and Wolfe's (2001) prediction, because there was only $50 \mathrm{msec}$ between each cuing event prior to the target. To account for this pattern of results, we assume that with rapid cues, as with longer cues, the inhibitory mechanism begins tagging the location as soon as attention is disengaged and moved to the next cued location. This inhibitory tag is the result of a process that takes a certain amount of time or number of stages to complete. The time to fully complete the inhibitory tag, however, appears to be slightly more than $50 \mathrm{msec}$, and therefore, there is some lag when rapid sequential cues are used. The result of this is that when attention is captured by the final fixation cue, the Cue 4 location (second to last location in the present experiments) has a more complete inhibitory tag relative to the last location, in which the inhibitory process has yet to fully develop. In addition, the decrease in IOR can be seen from Cue 4 back through Cue 1, although the overall magnitude of the effect is less than when more prolonged attentional dwell times occur between shifts of attention. Thus, in a typical visual search experiment in which there are multiple rapid shifts of attention, IOR does play a role, although this role appears to be limited to locations beyond the most recent previously attended location.

It is interesting that in the two experiments with long duration intervals between the offset of the final peripheral cue and the appearance of the target, dramatic differences were seen between the pattern of results in the short-cue conditions when the fixation cue was present and when it was absent. The results of Experiment 3 clearly indicate that under some circumstances, IOR can be found at several locations that were attended to only very briefly. The presence of the fixation cue, however, appears to stop the inhibitory tagging process, so that even with a long-duration fixation cue, IOR was not found for Cue 5 in Experiment 2. This is consistent with the findings of Pratt and Fischer (2002), who found no IOR when a 50 -msec peripheral cue was followed immediately by a $50-\mathrm{msec}$ fixation cue and then by a 100 -msec delay before the target. Moreover, Pratt and Fischer found IOR when the 50-msec peripheral cue was followed by a $50-\mathrm{msec}$ delay and then by the $50-\mathrm{msec}$ fixation cue and another 50-msec delay. Thus, a fixation event very quickly after the onset of a peripheral cue appears to stop the inhibitory tagging process, but this process can continue if attention is returned to fixation without such an event. In other words, stopping for a relatively short length of time at a location in visual search will allow for more robust IOR effects, including that at the most recent previously attended location. It is important to note that had the inhibitory tagging effect not begun immediately after attention was disengaged from each cued location in the present short-cue conditions, 
IOR would not have been observed at multiple locations with a delay between cue offset and target onset, as was the case in the short-cue condition in Experiment 3. We suggest that this delay allowed the inhibitory tagging process to be completed, but had the inhibitory tagging process been prevented (or interrupted) by rapid shifts of attention (as was suggested by Horowitz \& Wolfe, 2001), IOR would not have been observed at any cued location except Cue 5. Although it appears that IOR effects of the magnitude of 40-60 msec are not present in visual search, the present results demonstrate that inhibitory tagging of attended locations can begin to occur very rapidly and that IOR is present in visual search, although the process that creates the inhibitory tag requires a sufficient amount of time to be completed.

The present results have considerable practical application to visual search. Although we did not explicitly test for the presence of IOR in visual search (i.e., by using a typical visual search task), our results are consistent with those of others who have examined the role of both memory and IOR in visual search. For example, Gilchrist and Harvey (2000) investigated the role of memory in visual search by examining saccades during visual search for a target letter among a series of distractor letters, with a particular emphasis on refixations to previously attended locations. According to them, a perfect memory model would predict that individuals would never refixate on a previously attended location, whereas a memory-free model (such as that put forth by Horowitz \& Wolfe, 2001) would predict that refixations would occur at chance levels. The results fell between these two extremes, with individuals refixating on previously attended locations, but at a rate less than chance. Thus, Gilchrist and Harvey concluded that memory does play a limited role in visual search. Of particular importance to the present line of research, however, was their observation that there was evidence for IOR during visual search as judged by the number of fixations between an initial fixation on an item and a refixation on that same item; individuals very rarely refixated on a location that they had just attended. Thus, their finding are contrary to the predictions made by Horowitz and Wolfe (2001) but are consistent with the present research. A similar result has also been reported by Peterson, Kramer, Wang, Irwin, and McCarley (2001). Furthermore, Klein and MacInnes (1999) have provided evidence for IOR in visual search, using Where's Waldo displays and a subsequent probe detection task that required a fixation to the presence of a target. IOR was observed under this procedure also, since participants took longer to fixate a target that appeared in a general area that had been previously fixated. Collectively, these results suggest an interconnection between IOR, visual search, and memory processing.

In accordance with the idea that memory does operate in visual search, the present results also lend support to a memory-based model of IOR, in which previously attended locations are posited to be tagged and placed in memory so that attention does not return to these locations. Indeed, the notion that IOR is attributable to some form of memory is gaining empirical support. For example, Castel, Pratt, and Craik (2003) recently examined the effects of working memory load on IOR and observed no detriment to IOR when individuals had to remember how many odd digits were presented in the interval between the onsets of the cues and the detection targets. When this digit-monitoring task was replaced with a task that involved spatial working memory (such as remembering the number of upward-oriented arrows or objects), however, IOR was not found. This study provides empirical evidence to support the suggestions of Klein (2000) that IOR may rely on some form of spatial working memory.

In summary, the present experiments demonstrate that IOR can occur when attention is rapidly shifted to various locations, contrary to the suggestion of Horowitz and Wolfe (2001). In all of the present experiments, IOR was observed in some form when locations were rapidly cued in a random manner. Taken together with other research, the present findings suggest that shifts of attention have a memory component, even when several shifts are made in a rapid sequence. This adds support to the notion that both memory and IOR influence the manner in which we search the visual field.

\section{REFERENCES}

Abrams, R. A., \& Pratt, J. (1996). Spatially diffuse inhibition affects multiple locations: A reply to Tipper, Weaver, and Watson (1996). Journal of Experimental Psychology: Human Perception \& Performance, 22, 1294-1298.

Castel, A. D., Pratt, J., \& Craik, F. I. M. (2003). The role of spatial working memory in inhibition of return: Evidence from divided attention tasks. Perception \& Psychophysics, 65, 970-981.

Danziger, S., Kingstone, A., \& SNyder, J. J. (1998). Inhibition of return to successively stimulated locations in a sequential visual search paradigm. Journal of Experimental Psychology: Human Perception \& Performance, 24, 1467-1475.

Gilchrist, I. D., \& Harvey, M. (2000). Refixation frequency and memory mechanisms in visual search. Current Biology, 10, 1209-1212.

Horowitz, T. S., \& Wolfe, J. M. (1998). Visual search has no memory. Nature, 394, 575-577.

Horowitz, T. S., \& Wolfe, J. M. (2001). Search for multiple targets: Remember the targets, forget the search. Perception \& Psychophysics, 63, 272-285.

KLEIN, R. M. (1988). Inhibitory tagging system facilitates visual search. Nature, 334, 430-431.

KLEIN, R. M. (2000). Inhibition of return. Trends in Cognitive Sciences, 4, 138-147.

KLEIN, R. M., \& MacInnes, W. J. (1999). Inhibition of return is a foraging facilitator in visual search. Psychological Science, 10, 346-352.

Müller, H. J., \& VON MüHLEnen, A. (2000). Probing distractor inhibition in visual search: Inhibition of return. Journal of Experimental Psychology: Human Perception \& Performance, 26, 1591-1605.

Peterson, M. S., Kramer, A. F., Wang, R. F., Irwin, D. E., \& McCarley, J. S. (2001). Visual search has memory. Psychological Science, 12, 287-292.

Posner, M. I., \& Cohen, Y. (1984). Components of visual orienting. In H. Bouma \& D. G. Bouwhuis (Eds.), Attention and performance X: Control of language processes (pp. 531-556). Hillsdale, NJ: Erlbaum.

Pratt, J., \& ABrams, R. A. (1995). Inhibition of return to successively cued spatial locations. Journal of Experimental Psychology: Human Perception \& Performance, 21, 1343-1353. 
Pratt, J., \& Fischer, M. (2002). Examining the role of the fixation cue in inhibition of return. Canadian Journal of Experimental Psychology, 56, 294-301.

Pratt, J., Kingstone, A., \& Khoe, W. (1997). Inhibition of return in location- and identity-based choice decision tasks. Perception \& Psychophysics, 59, 964-971.

SNYDER, J. J., \& Kingstone, A. (2000). Inhibition of return and visual search: How many separate loci are inhibited? Perception \& Psychophysics, 62, 452-458.

SNYDER, J. J., \& KINGSTONE, A. (2001). Inhibition of return at multiple locations in visual search: When you see it and when you don't. Quarterly Journal of Experimental Psychology, 54A, 1221-1237.

TAKedA, Y., \& YAGI, A. (2000). Inhibitory tagging in visual search can be found if search stimuli remain visible. Perception \& Psychophysics, 62, 927-934.

Tipper, S. P., Weaver, B., Jerreat, L. M., \& Burak, A. L. (1994).
Object- and environment-based inhibition of return of visual attention. Journal of Experimental Psychology: Human Perception \& Performance, 20, 478-499.

Tipper, S. P., Weaver, B., \& Watson, F. L. (1996). Inhibition of return to successively cued spatial locations: Commentary on Pratt and Abrams (1995). Journal of Experimental Psychology: Human Perception \& Performance, 22, 1289-1293.

Treisman, A. (1986). Features and objects in visual processing. Scientific American, 254, 114-125.

Treisman, A., \& Gelade, G. (1980). A feature integration theory of attention. Cognitive Psychology, 12, 97-136.

Wolfe, J. M., \& PoKorny, C. W. (1990). Inhibitory tagging in visual search: A failure to replicate. Perception \& Psychophysics, 48, 357-362.

(Manuscript received November 5, 2001; revision accepted for publication March 21, 2003.) 